\title{
Modeling light propagation through bacterial colonies and its correlation with forward scattering patterns
}

\author{
Euiwon Bae \\ Nan Bai \\ Purdue University \\ School of Mechanical Engineering \\ West Lafayette, Indiana 47906
}

\author{
Amornrat Aroonnual \\ Purdue University \\ Department of Food Science \\ Molecular Food Microbiology Laboratory \\ West Lafayette, Indiana 47906
}

\author{
J. Paul Robinson \\ Purdue University \\ Department of Basic Medical Science \\ and \\ Weldon School of Biomedical Engineering \\ West Lafayette, Indiana 47906
}

\author{
Arun K. Bhunia \\ Purdue University \\ Department of Food Science \\ Molecular Food Microbiology Laboratory \\ West Lafayette, Indiana 47906
}

\section{E. Daniel Hirleman}

Purdue University

School of Mechanical Engineering

West Lafayette, Indiana 47906

\begin{abstract}
Bacterial colonies play an important role in the isolation and identification of bacterial species, and plating on a petri dish is still regarded as the gold standard for confirming the cause of an outbreak situation. A bacterial colony consists of millions of densely packed individual bacteria along with matrices such as extracellular materials. When a laser is directed through a colony, complicated structures encode their characteristic signatures, which results in unique forward scattering patterns. We investigate the connection between the morphological parameters of a bacterial colony and corresponding forward scattering patterns to understand bacterial growth morphology. A colony elevation is modeled with a Gaussian profile, which is defined with two critical parameters: center thickness and diameter. Then, applying the scalar diffraction theory, we compute an amplitude modulation via light attenuation from multiple layers of bacteria while a phase modulation is computed from the colony profile. Computational results indicate that center thickness plays a critical role in the total number of diffraction rings while the magnitude of the slope of a colony determines the maximum diffraction angle. Experimental validation is performed by capturing the scattering patterns, monitoring colony diameters via phase contrast microscope, and acquiring the colony profiles via confocal displacement meter. (C) 2010 Society of Photo-Optical Instrumentation Engineers. [DOI: 10.1117/1.3463003]
\end{abstract}

Keywords: bacterial colony; diffraction; phase modulation.

Paper 10056RR received Feb. 2, 2010; revised manuscript received May 7, 2010; accepted for publication May 12, 2010; published online Jul. 16, 2010.

\section{Introduction}

Pathogenic bacteria have caused numerous infectious diseases in host living organisms either by contact or food consumption. Recent outbreaks from numerous food sources such as tomatoes and peanut butter remind us of the vulnerability of the food supply chain to contamination with pathogenic bacteria. The gold standard to detect and isolate a bacterial species is to grow colonies in a petri dish. A bacteria colony is defined as a group of individual bacterium $\left(\sim 10^{8}\right)$ compactly packed together to form an entity different from a surrounding environment. A colony consists of not only the bacteria itself but also extracellular materials, which are secreted as the colony grows. This combined structure can be analyzed to provide distinguishable phenotypic characteristics with proper interrogation methods. For example, differences of color, size, and shape can be utilized as distinguishing traces of a certain bacteria species under certain growth conditions. However, a selective medium that allows only certain types of species to

Address all correspondence to: Euiwon Bae, School of Mechanical Engineering, Purdue University, West Lafayette, IN 47906. Tel: 765-494-4762; Fax: 765-4940539; E-mail: ebae@purdue.edu grow and substantially suppresses the growth of others limits applicability to a small number of bacterial species. Therefore, it is advantageous to use nonselective media, which can grow various species on the same media. When different bacteria species are grown in this general media, their phenotypic characteristics tend to be quite similar, which renders morphological differentiation challenging.

Recently, a group of researchers reported a novel method of applying a forward scattering technique to identify a bacterial colony with highly accurate classification and broad applicability. The proposed technology, called BARDOT (bacteria rapid detection using optical scattering technology), provided highly repeatable and distinguishable forward scattering patterns all the way down to strain levels for some of the test cases. The previous research provided the theoretical modeling using scalar diffraction theory, ${ }^{1,2}$ experimental verification for time-resolved scattering, ${ }^{3}$ and application to the actual food samples. ${ }^{4}$ In life science, automated bacterial colony counting is a topic of interest and various image processing techniques have been suggested for optimal perfor-

$1083-3668 / 2010 / 15(4) / 045001 / 11 / \$ 25.00$ (C) 2010 SPIE 
mance considering the optical response characteristics of the colony. ${ }^{5-9}$ Several authors have modeled the profile of the bacterial colony elevation as a convex shape with different radii of curvature, ${ }^{1}$ a thin film with a decreasing tailing edge, ${ }^{5}$ and a Gaussian profile. ${ }^{6}$ The results of our most recent study on the smaller bacterial colony profile indicate that a Gaussian profile is quite satisfactory for describing the actual profile of the bacterial colony. ${ }^{10}$ Therefore, we model a bacterial colony as an optical amplitude/phase modulator with a Gaussian cross section in our study.

Similar ring-type diffraction patterns were observed from previous research involving neumatic liquid crystals (LCs), where the patterns were due to the laser-induced self-phase modulation phenomenon. ${ }^{11-20}$ LC molecules were rotated in proportion to the intensity of the incident laser, which results in refractive index variations with a Gaussian profile. When this laser passes through an LC film of several hundred micrometer thickness, the optical wavefront is modulated to create a Gaussian wavefront that is propagated to a screen to create several diffraction rings. Some researchers investigated the field curvature effect on the diffraction rings, ${ }^{16,17}$ while others worked on the relationship between Fredericksz transitions and primary/secondary diffraction rings. ${ }^{11-14,20}$

Although the fundamental idea was revealed, more quantitative analysis is required to understand the morphological parameters of the bacterial colony and their correlation to the forward scattering pattern. Since the wavefront after the bacterial colony combines the incoming wavefront with the depth-averaged effect of a phase modulator, we designate some important morphological parameters and analyze their correlations to the far-field scattering patterns. Section 2 introduces theoretical modeling of light colony interactions and experimental procedures. Section 3 provides the simulation results when diameter and colony height are varied independently as well as experimental measurements of scattering patterns, colony diameters, and profiles. Section 4 discusses the validation and correlation between the scattering pattern and colony morphology.

\section{Materials and Methods}

\subsection{Modeling Light-Colony Interactions}

\subsubsection{Derivation of diffraction modeling}

We define the coordinate systems for the source, the colony, and the imaging coordinate as $x_{s}, y_{s} ; x_{a}, y_{a}$; and $x_{i}, y_{i}$, respectively, as shown in Fig. 1(c). Assuming a TEM 00 mode of laser beam centered on the $z$ axis, electric field on the aperture plane $E_{1}$ can be expressed as ${ }^{1}$

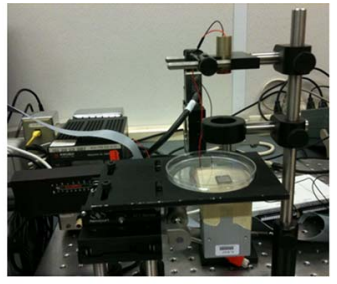

(a)

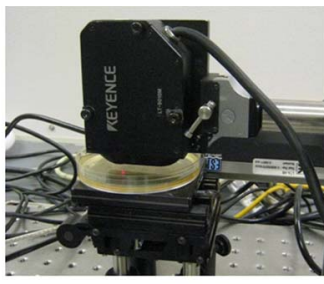

(b)

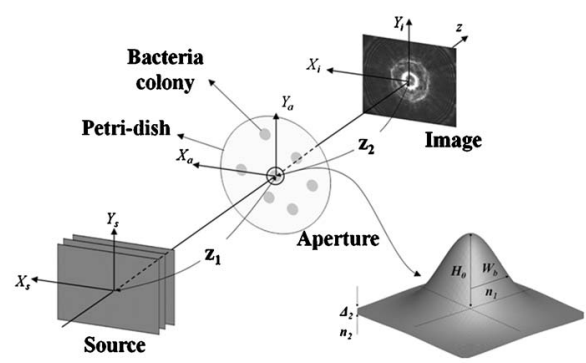

(c)

Fig. 1 Experimental setup and definition of coordinate system: (a) forward scatterometer with laser diode (635 nm); (b) confocal displacement measurement (CDM) system with 2-D rastering via $X Y$ stage system; and (c) coordinate system for source, aperture, and image coordinates and the bacterial colony morphology fitted with Gaussian shape. Here $H_{0}$ is the center thickness, $w_{b}$ is the $1 /$ e radius, $n_{1}$ and $n_{2}$ are the refractive indices for colony and agar, and $\Delta_{2}$ represents the agar thickness.

$$
\begin{aligned}
E_{1}\left(x_{a}, y_{a}, z_{1}\right)= & E_{0} \exp [ \\
& \left.-\frac{\left(x_{a}^{2}+y_{a}^{2}\right)}{w^{2}\left(z_{1}\right)}\right] \exp \left(i k z_{1}\right) \exp \left[i k \frac{\left(x_{a}^{2}+y_{a}^{2}\right)}{2 R\left(z_{1}\right)}\right],
\end{aligned}
$$

where $E_{0}$ is the on-axis field strength; three terms account for variations of amplitude of field, longitudinal phase, and radial phase, respectively; $x_{a}$ and $y_{a}$ are the coordinates in the aperture; and $w\left(z_{1}\right)$ and $R\left(z_{1}\right)$ are the beam waist and radius of the wavefront in the colony plane $\left(z=z_{1}\right)$, which is defined by

$$
w^{2}(z)=w_{0}^{2}\left[1+\left(\frac{z}{z_{0}}\right)^{2}\right], \quad R(z)=z\left[1+\left(\frac{z_{0}}{z}\right)^{2}\right],
$$

where $z_{0}$ is defined as the $z$ location where $1 / e^{2}$ radius has expanded to $\sqrt{2}$ times of beam waist $w_{0}$. Then, we apply the Huygens-Fresnel principle in rectangular coordinates. Using Eq. (1), the electric field $E_{2}$ at the imaging plane can be expressed as

$$
E_{2}\left(x_{i}, y_{i}\right)=\frac{1}{i \lambda} \iint_{\Sigma} t\left(x_{a}, y_{a}\right) E_{1}\left(x_{a}, y_{a}\right) \exp \left\{i k\left[\phi\left(x_{a}, y_{a}\right)\right]\right\} \exp \left(\frac{i k r_{a i}}{r_{a i}}\right) \cos \theta \mathrm{d} x_{a} \mathrm{~d} y_{a}
$$


where $x_{i}$ and $y_{i}$ are points on the image plane, $\Sigma$ denotes the colony surface, $t\left(x_{a}, y_{a}\right)$ is the 2-D transmission coefficient, $\phi\left(x_{a}, y_{a}\right)$ is the 2-D phase modulation factor, $r_{a i}$ is the distance from the aperture plane to the image plane, $\lambda$ is the wavelength, and $n_{2}$ and $\Delta_{2}$ represent the refractive index and thickness of Brain heart infusion (BHI) agar, respectively. Based on the scalar diffraction theory, the diffracted field on the image coordinates can be formulated via Fresnel approximation as

$$
\begin{aligned}
E_{2}\left(x_{i}, y_{i}\right) \approx & C_{1} \iint_{\Sigma} t\left(x_{a}, y_{a}\right) \exp [ \\
& \left.-\frac{\left(x_{a}^{2}+y_{a}^{2}\right)}{w^{2}\left(z_{1}\right)}\right] \exp \left[i k \frac{\left(x_{a}^{2}+y_{a}^{2}\right)}{2 R\left(z_{1}\right)}\right] \exp \left[i k \frac{\left(x_{a}^{2}+y_{a}^{2}\right)}{2 z_{2}}\right] \\
& \times \exp \left[i k \phi\left(x_{a}, y_{a}\right)\right] \exp \left[-i 2 \pi\left(f_{x} x_{a}+f_{y} y_{a}\right)\right] \mathrm{d} x_{a} \mathrm{~d} y_{a},
\end{aligned}
$$

where $f_{x}$ and $f_{y}$ are defined as $f_{x}=x_{i} / \lambda z_{2}$ and $f_{y}=y_{i} / \lambda z_{2}$. When Eq. (2) is rearranged, the constants and independent variables are consolidated as

$$
C_{1}=\frac{E_{0} \exp \left(i k n_{2} \Delta_{2}\right) \exp \left[i k\left(z_{1}+z_{2}\right)\right] \exp \left[\left(i k / 2 z_{2}\right)\left(x_{i}^{2}+y_{i}^{2}\right)\right]}{i \lambda z_{2}},
$$

where $k$ is the wave number. Detailed assumptions and the derivation are shown elsewhere. ${ }^{1}$

\subsubsection{Amplitude component}

Our scanning electron microscopy (SEM) observation revealed both microscopic and macroscopic details of how millions of individual bacteria were packed to form a colony. ${ }^{2}$ The results indicated that the individual bacterium tended to form a layered structure rather than a distribution of randomly oriented bacterium, which provided for us a ground on which to model the light-bacteria interactions using a layer model as a first-order approximation. We divided the bacterial colony profile as multiple layers of bacteria with a thickness of $\Delta \mu \mathrm{m}$, considering the physical dimensions of the individual bacteria and extracellular materials. Although the reduction in transmitted light can originate from both reflections and absorptions, we assume the normal incident reflection is the major contributor of field loss to our layer model based on the interrogating wavelength and refractive index of a bacterium. With respect to this assumption, Fig. 2 shows the field attenuated for the $k$ 'th layer of bacterium, which can be modeled as

$$
E_{k+1}=E_{k}\left(1-r_{k}\right),
$$

where $r_{k}$ is the reflection coefficient for the $k^{\prime}$ th layer and is assumed to be constant for all the layers. Considering all the multiple layers in the colony, the overall 2-D transmission coefficient is defined as

$$
t\left(x_{a}, y_{a}\right)=\frac{E_{\text {out }}}{E_{0}}=\left(1-r_{1}\right)\left(1-r_{k}\right)^{2 l}\left(1-r_{2}\right),
$$

where the exponent $l$ is defined with the colony profile $H\left(x_{a}, y_{a}\right)$, and the layer thickness $\Delta$ as

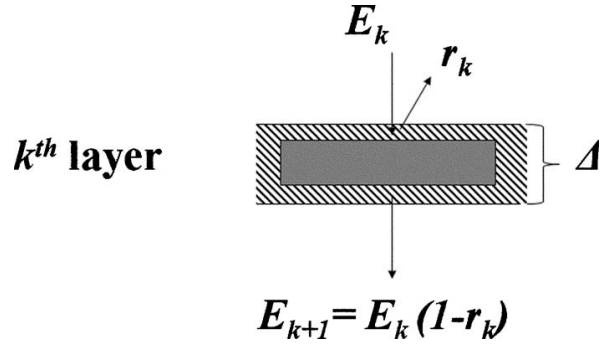

Fig. 2 Schematic diagram for amplitude modulation model via multiple layers, where the rectangular box and the shaded box represent bacteria and extracellular material: $E_{k}$ denotes the electric field incident on the $k$ th layer of bacteria exiting as $E_{k+1}$. The loss is modeled via the reflection coefficient $r_{k}$ with the layer (bacterium + extracellular material) thickness as $\Delta$.

$$
l=H\left(x_{a}, y_{a}\right) / \Delta .
$$

The different reflection coefficients $r_{k}, r_{1}$, and $r_{2}$ are defined since $r_{k}$ represents the interbacterium reflection, while $r_{1}$ and $r_{2}$ model the reflection between air-bacterium and bacteriumagar. This can be modeled as

$$
r_{k}=\left|\frac{n_{1}-n_{\mathrm{ec}}}{n_{1}+n_{\mathrm{ec}}}\right|, \quad r_{1}=\left|\frac{n_{0}-n_{1}}{n_{0}+n_{1}}\right|, \quad r_{2}=\left|\frac{n_{1}-n_{2}}{n_{1}+n_{2}}\right|,
$$

where $n_{0}, n_{1}, n_{2}$, and $n_{\mathrm{ec}}$ are the refractive indices for air, bacteria, agar, and extracellular materials. Since our scalar diffraction theory does not consider the polarization effect, we simply model the attenuation from the thickness of the colony and take the absolute value of the reflection coefficients.

\subsubsection{Phase component}

The phase component of the diffraction integral is governed by the optical path length term

$$
\phi\left(x_{a}, y_{a}\right)=n_{1} H\left(x_{a}, y_{a}\right)+\left[H_{0}-H\left(x_{a}, y_{a}\right)\right],
$$

where $H_{0}$ and $H\left(x_{a}, y_{a}\right)$ are the center thickness and a profile of the bacterial colony. In addition, we define the effective diameter $D$ of the Gaussian profile colony as

$$
D=2 w_{b} F,
$$

where $w_{b}$ is the $1 / e$ radius of a Gaussian profile, and $F$ is the factor (1.6) multiplied to render a $1 / e^{3}$ radius. Equation (10) can be further rearranged when we assume the Gaussian profile of the bacterial colony as ${ }^{21}$

$$
\phi\left(x_{a}, y_{a}\right)=\left(n_{1}-1\right) H_{0} \exp \left[-\frac{\left(x_{a}^{2}+y_{a}^{2}\right)}{w_{b}^{2}}\right]+H_{0}
$$

Therefore, the overall phase modulation $\phi^{\prime}\left(x_{a}, y_{a}\right)$ is contributed from the radial phase and the bacterial phase modulation, which is formulated as 
Table 1 Common simulation parameters.

\begin{tabular}{lccc}
\hline Source & $Z_{1}$ & 100 & $\mathrm{~mm}$ \\
& $w$ & 0.5 & $\mathrm{~mm}$ \\
& $\lambda$ & 0.635 & $\mu \mathrm{m}$ \\
Colony & $P$ & 1 & $\mathrm{~mW}$ \\
& $Z_{2}$ & 30 & $\mathrm{~mm}$ \\
& $\Delta$ & 2 & $\mu \mathrm{m}$ \\
Image & $n_{1}$ & 1.38 & - \\
\hline & $n_{2}$ & 1.33 & - \\
& $n_{\mathrm{ec}}$ & 1.35 & - \\
\hline
\end{tabular}

$$
\begin{aligned}
E_{2}\left(x_{i}, y_{i}\right) \approx & C_{2} \iint_{\Sigma} t\left(x_{a}, y_{a}\right) \exp [ \\
& \left.-\frac{\left(x_{a}^{2}+y_{a}^{2}\right)}{w^{2}\left(z_{1}\right)}\right] \exp \left[i k \phi^{\prime}\left(x_{a}, y_{a}\right)\right] \times \exp \left[-i 2 \pi\left(f_{x} x_{a}\right.\right. \\
& \left.\left.+f_{y} y_{a}\right)\right] \mathrm{d} x_{a} \mathrm{~d} y_{a}, \\
\phi^{\prime}\left(x_{a}, y_{a}\right)= & \left(\frac{x_{a}^{2}+y_{a}^{2}}{2}\right)\left[\frac{1}{R\left(z_{1}\right)}+\frac{1}{z_{2}}\right]+\left(n_{1}-1\right) H_{0} \exp [ \\
& \left.-\frac{\left(x_{a}^{2}+y_{a}^{2}\right)}{w_{b}^{2}}\right]
\end{aligned}
$$

where $C_{2}$ is a constant $C_{1}$ multiplied by $\exp \left(i k H_{0}\right)$.

Then, the intensity of the electric field (power) is computed via

$$
I=\frac{1}{2} c \varepsilon\left|E_{2}\right|^{2}
$$

where $c$ is the speed of light in a vacuum, and $\varepsilon$ is the permittivity. We then computed the diffraction pattern at the far field according to the parameters defined in Table 1.

\subsection{Experiments}

\subsubsection{Sample preparation}

Salmonella montevideo cultures were selected to be inoculated in BHI broth and incubated at $37{ }^{\circ} \mathrm{C}$ overnight. The culture was then diluted (10-fold dilution) in phosphate buffer saline (PBS). We spread $100 \mu \mathrm{l}$ of the appropriate concentration thoroughly on the BHI agar and cultivated at $37{ }^{\circ} \mathrm{C}$ in the incubator. At the specified growth time, the plate was taken to BARDOT for measurement, which were $6.5 \mathrm{~h}$ (diameter around $90 \mu \mathrm{m}), 7 \mathrm{~h}(120 \mu \mathrm{m}), 8 \mathrm{~h}(160 \mu \mathrm{m})$, and $9 \mathrm{~h}(300 \mu \mathrm{m})$.

\subsubsection{Forward scatterometer}

A forward scatterometer [Fig. 1(a)] was built using (1) a 635-nm diode laser (Coherent 0221-698-01 REV B, California) with $1 \mathrm{~mW}$ of power and a 1-mm diameter; (2) a single biconvex lens (BK7 H32-717, Edmund Optics, New Jersey) with a focal length of $150 \mathrm{~mm}$; (3) a monochromatic IEEE1394 CMOS array (PixeLINK, Ottawa, Ontario, Canada) with $1280 \times 1024$ resolution and $6.7 \times 6.7 \mu \mathrm{m}^{2}$ for each pixel, used as the imaging sensor; and (4) an $X Y$ linear translation stage (two of 850-HS, Newport, New York) and a multiaxis closed-loop controller (ESP300, Newport, New York) to move the plate in the $x-y$ plane to align the laser with the center of each selected bacterial colony. The biconvex lens was used to control the beam diameter similar to the bacterial colony diameter via controlling the beam waist within the Raleigh range of the focused Gaussian beam. The focusing beam diameter $d$ is computed via ${ }^{22}$

$$
d=2 \omega_{02}=\frac{2 f \lambda}{\pi \omega_{01}}
$$

where $\omega_{02}$ is the beam waist of the focusing beam, $f$ is the focal length of the lens, $\lambda$ is the wavelength of the laser beam, and $\omega_{01}$ is the radius of the incoming Gaussian beam. The depth of focus, where the bacterial colony should be located, is two times the Raleigh range $z_{R}$ of the focused laser beam;

$$
2 z_{R}=\frac{2 \pi \omega_{02}^{2}}{\lambda} .
$$

The calculated depth of focus for the current optical system configuration was around $36 \mathrm{~mm}$.

\subsubsection{Phase contrast microscopy and confocal displacement meter}

The diameters of the bacterial colonies were measured by a phase contrast microscope (Leica Microsystems, Illinois). The objective was $\times 10$ and the phase contrast image mode was used to image and measure the diameter of the colonies. Even though the phase contrast microscope provided qualitative phase information, it did not correlate the image with the colony profile quantitatively. Therefore, a confocal displacement meter (Keyence LT9010, New Jersey) was integrated with the $X-Y$ motorized stage to obtain the height profile of each individual bacterial colony [Fig. 1(b)]. We tested conventional laser triangular sensors to measure the profile, but the complexity of the sample (diffuse reflectivity, transparency, and multiple surface reflections) resulted in an unreliable shape profile (data not shown). The CDM instrument implements a "confocal principle" in the laser triangulation technique to deal with these issues and provided highly reliable surface scanning method by using a 670-nm laser light source with the spot size of $2 \mu \mathrm{m}$ and the vertical reference distance of $6 \mathrm{~mm}$ and a scanning resolution up to ${ }^{23} 0.01 \mu \mathrm{m}$.

\section{Results}

We theoretically investigated the effect of morphological variation of the bacterial colonies to diffraction patterns on the imaging plane and measured the actual bacterial colony morphologies via microscope and profilometer. To decouple the 


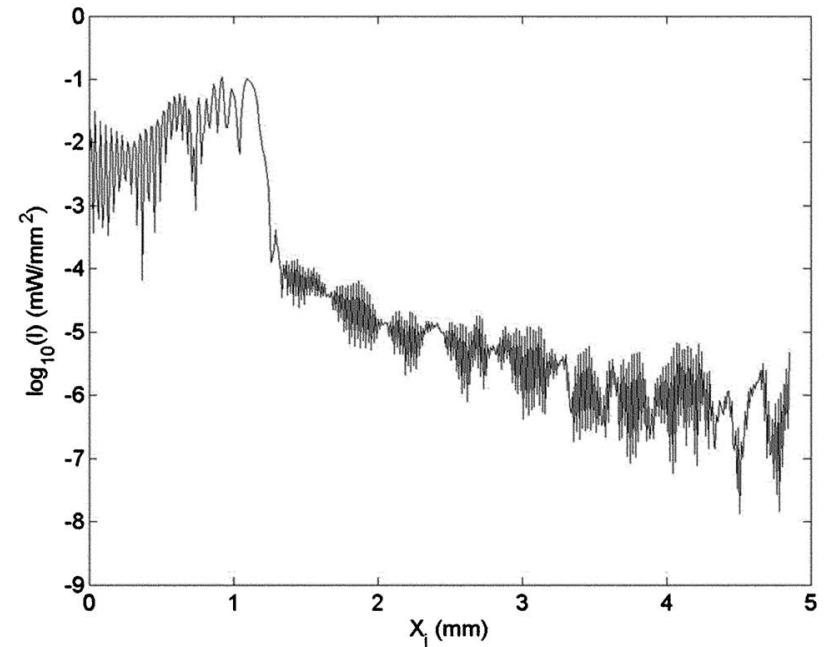

(a)

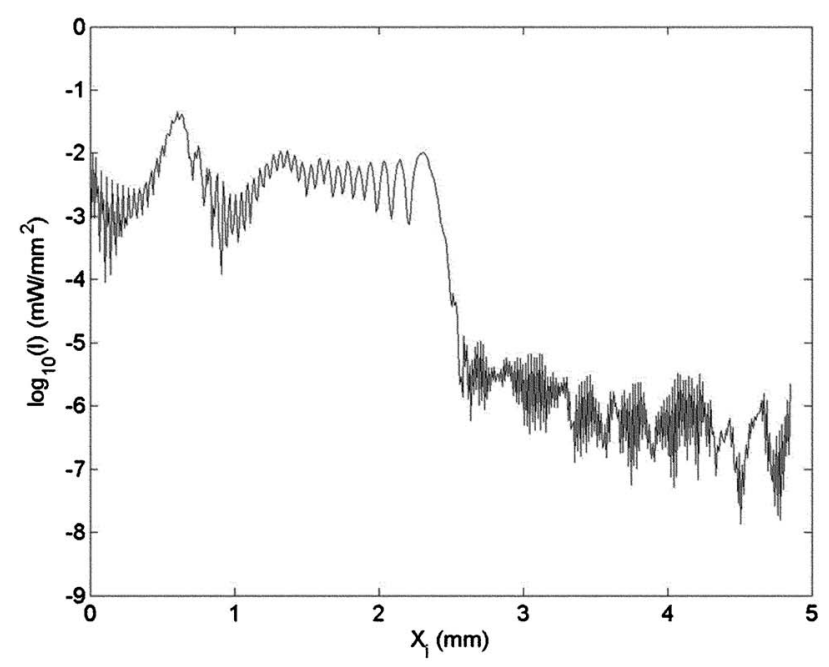

(c)

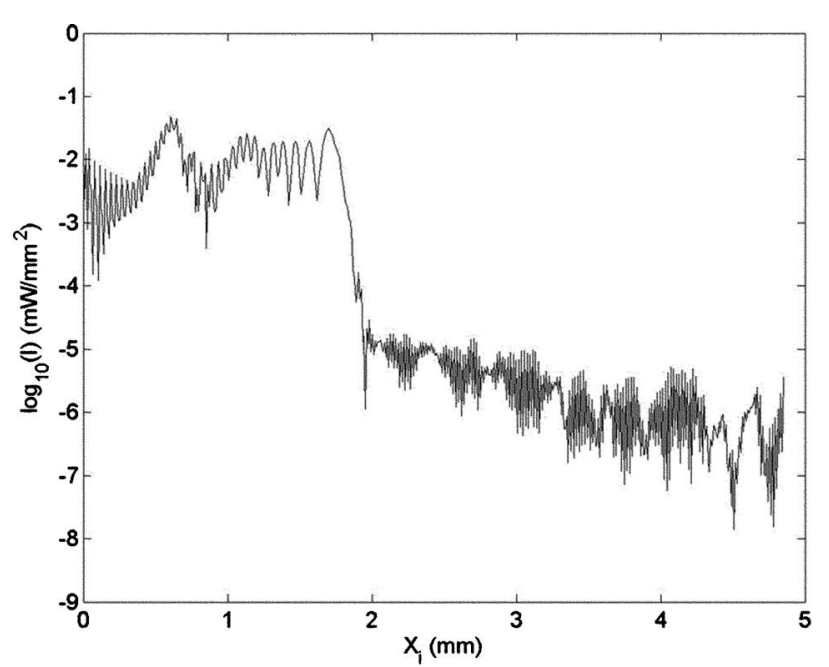

(b)

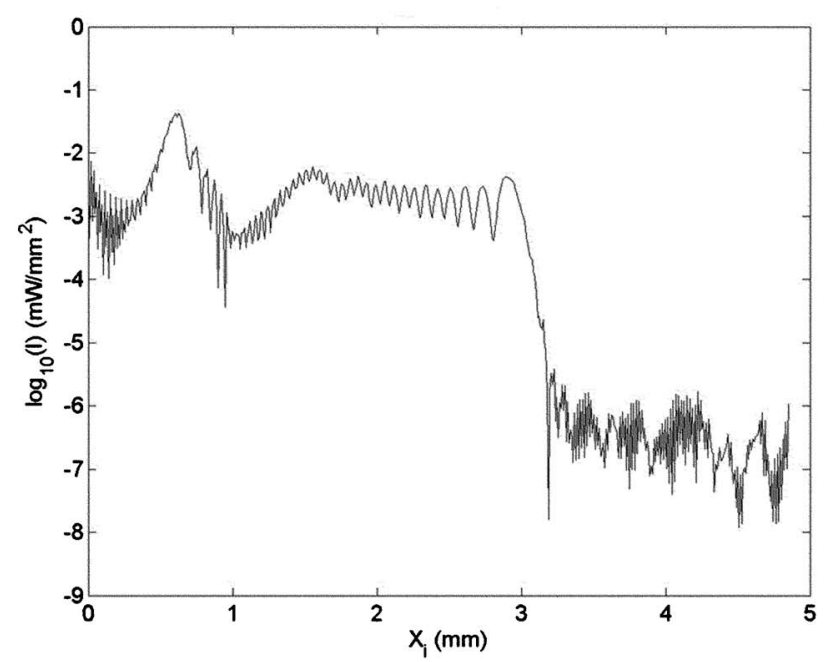

(d)

Fig. 3 Forward scattering pattern for center thickness $\left(H_{0}\right)$ variation when diameter is kept constant: $H_{0}=(\mathrm{a}) 0.03,(\mathrm{~b}) 0.05,(\mathrm{c}) 0.07$, and $(\mathrm{d})$ $0.09 \mathrm{~mm}$. The number of peaks and maximum scattering angle increases as $H_{0}$ increases.

effect of morphological parameters, we computed the colony center thickness effect and the colony diameter effect separately.

\subsection{Height Variation versus Diffraction Pattern}

The diameter of the bacterial colony $D$ was kept constant at $1.024 \mathrm{~mm}$ and the center thickness $H_{0}$ of the bacterial colony was modified from 10 to $110 \mu \mathrm{m}$ in 5 - $\mu \mathrm{m}$ steps. The maximum thickness was adapted from the previously published experimental results from the confocal microscopy. ${ }^{1}$ The corresponding profile was modified with Eqs. (3)-(15) and their respective diffraction patterns were displayed for radii of 0 to $5 \mathrm{~mm}$ due to their circularly symmetric patterns. Since the dynamic range of the diffraction pattern varied significantly, we plotted the logarithm of the intensity (in milliwatts per square millimeters). Figure 3 shows the result for $H_{0}$ for 30, 50, 70, and $90 \mu \mathrm{m}$. The computational results indicated the following two observations:
1. When the $H_{0}$ value increased for a constant diameter, the maximum diffraction angle increased.

2. When the $H_{0}$ value increased for a constant diameter, the total number of rings also increased.

For example, for $H_{0}=30 \mu \mathrm{m}$, the maximum half diffraction angle was $2.13 \mathrm{deg}$, while the $H_{0}=90 \mu \mathrm{m}$ resulted in $5.71 \mathrm{deg}$ (with an intensity cutoff value of 5 $\times 10^{-5} \mathrm{~mW} / \mathrm{mm}^{2}$ ). Meanwhile, the total numbers of rings (peaks) were counted as 28 and 65, respectively. Note also that the intensity level of the region between $X_{i}=0$ and $X_{i}$ $=0.5$ monotonically decreased as $H_{0}$ increased, while the location of the first peak did not vary significantly.

\subsection{Diameter Variation versus Diffraction Pattern}

In this study, we kept the $H_{0}$ value of the bacterial colony as $50 \mu \mathrm{m}$ and investigated the effect of the $D$ variations when it was varied from 0.384 to $1.05 \mathrm{~mm}$ in $10-\mu \mathrm{m}$ steps. The corresponding profile was also modified with Eqs. (3)-(15) and 


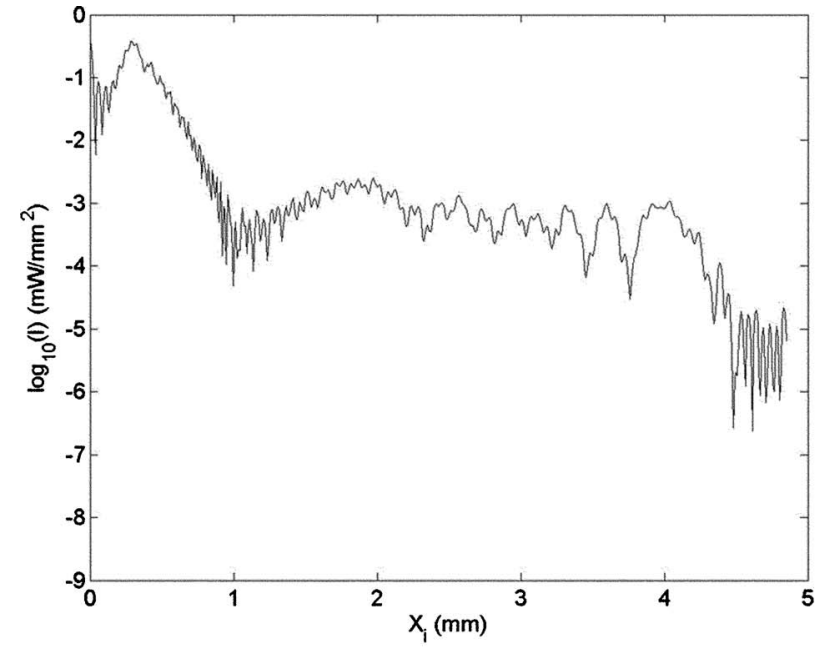

(a)

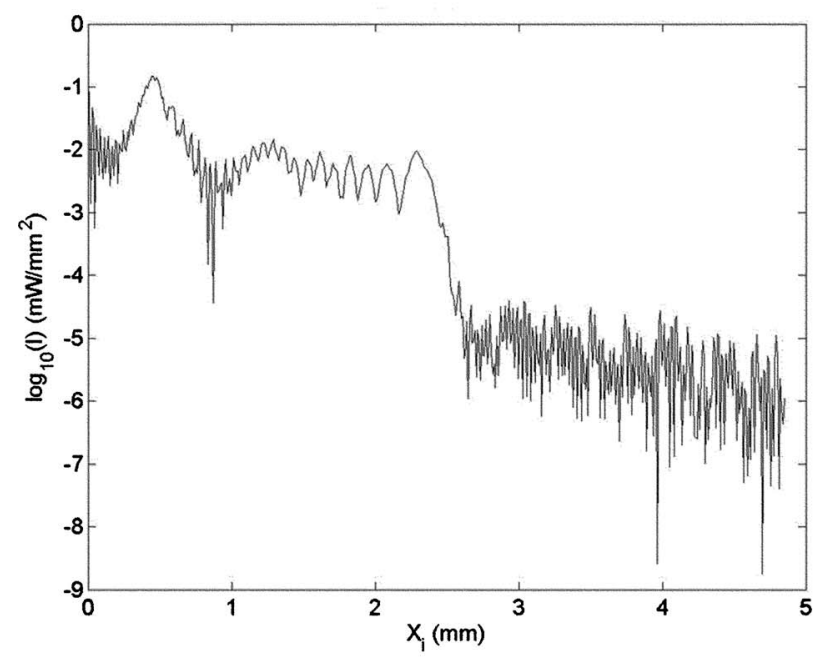

(c)

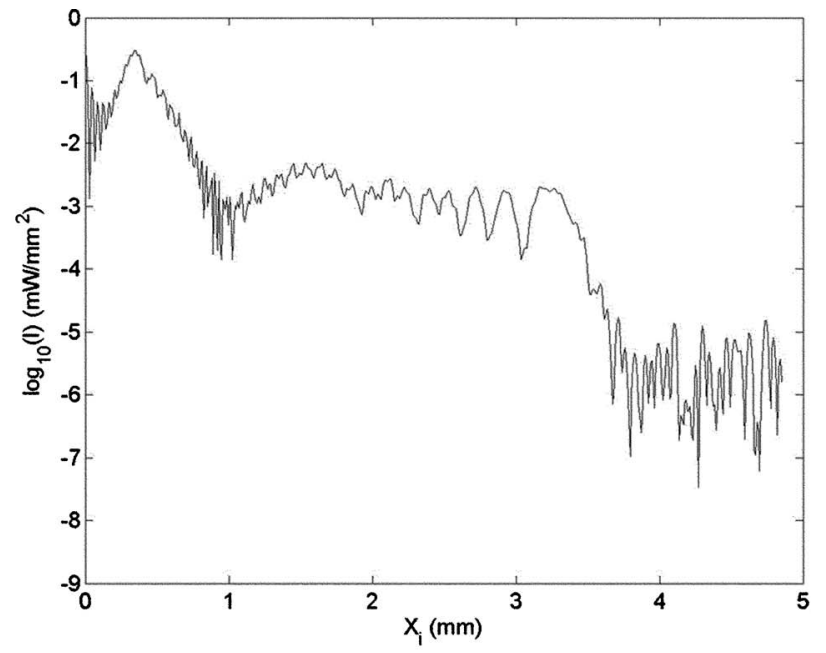

(b)

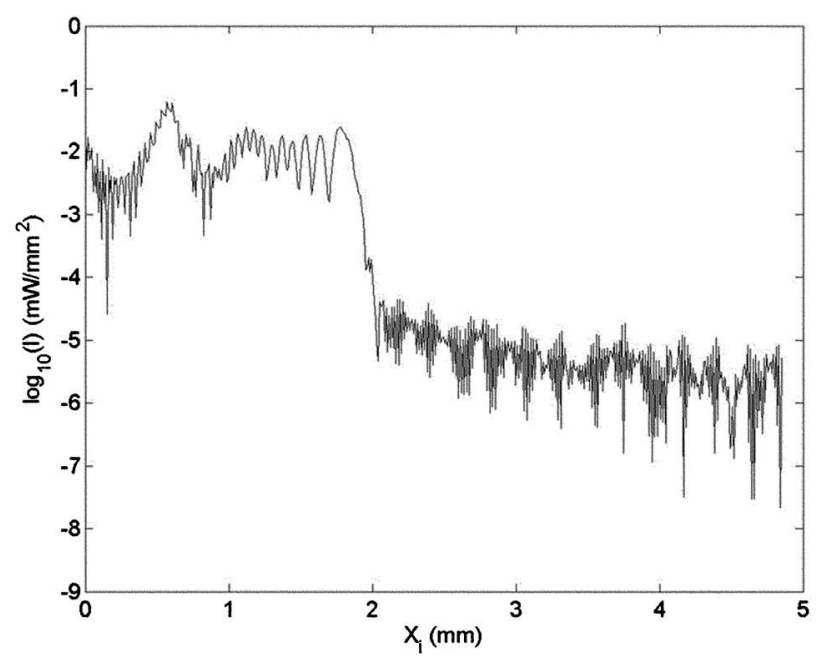

(d)

Fig. 4 Forward scattering pattern for colony diameter variation when $H_{0}$ is kept constant and $D$ is (a) 0.384 , (b) 0.48 , (c) 0.704 , and (d) 0.96 mm. Note that the maximum scattering angle decreases as $D$ increases.

their respective diffraction patterns are displayed in Fig. 4 for the colony diameters of $0.384,0.48,0.704$, and $0.96 \mathrm{~mm}$. The diffraction calculation provided the following understandings:

1. When the center thickness was kept constant, the maximum diffraction angle was inversely proportional to the colony diameter.

2. When the center thickness was kept constant, the total number of rings was also kept constant irrespective of the colony diameter variation.

For example, the maximum half diffraction for a diameter of $0.384 \mathrm{~mm}$ was $7.676 \mathrm{deg}$, while for a diameter of $0.96 \mathrm{~mm}$, it was only $3.676 \mathrm{deg}$. The total number of rings did not change much once the $H_{0}$ was kept constant. Compared to the results of Sec. 3.1, the first peak location translated outward and peak value decreased as the $D$ increased.

\subsection{Correlation between Morphology and Diffraction Patterns}

To understand the correlation between the colony morphology and the diffraction patterns, we repeated the calculation for different $D$ (Sec. 3.1) and different $H_{0}$ (Sec. 3.2). Figure 5(a) shows the relationship between the $H_{0}$ and the total number of peaks (diffraction rings) when $D$ was varied for $D=0.768$, 0.896 , and $1.024 \mathrm{~mm}$. The result clearly indicated that the $H_{0}$ value was the major factor contributing to the total number of diffraction rings observed at the imaging plane irrespective of the $D$ value. Figure 5(b) indicates the relationship between the colony diameter and the total number of peaks, which remained relatively constant across the diameter but increased as the $H_{0}$ values increased, which confirmed the result from Fig. 5(a).

Figure 6 displays the phase [Fig. 6(a)] and the first derivative of the phase [Fig. 6(b)] for a diameter of $1.024 \mathrm{~mm}$ and $H_{0}$ values of 30,50 , and $70 \mu \mathrm{m}$. Since the center thicknesses are different, the phase lag from $70 \mu \mathrm{m}$ is approximately 2.5 times as great as that from a $30-\mu \mathrm{m}$ thickness, as shown in Fig. 6(a). The derivative of the phase in Fig. 6(b) for the three thicknesses shows the minima at the $X_{i}=0.23 \mathrm{~mm}$ location since this is the deflection point of the phase in Fig. 6(a). The 


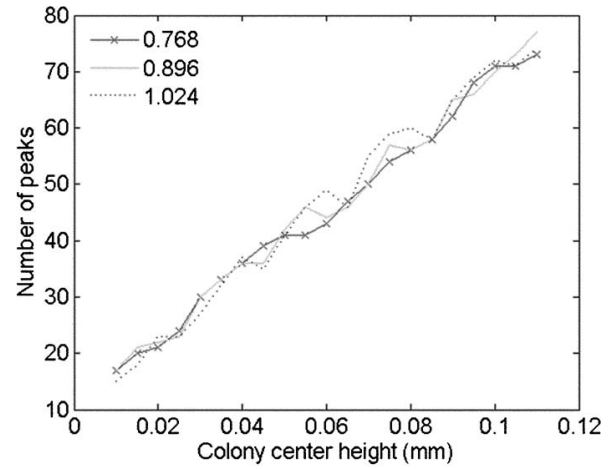

(a)

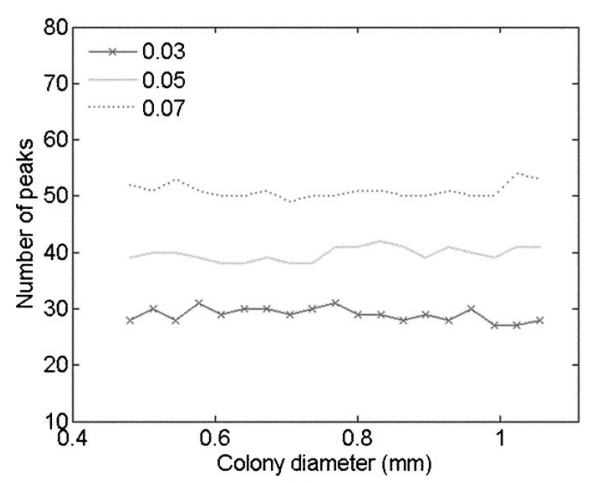

(b)

Fig. 5 Correlation of the number of rings when (a) colony thickness and (b) colony diameter were varied.

magnitude of the derivative of the wavefront for $70 \mu \mathrm{m}$ shows the largest value, which corresponded to the half angle of $4.42 \mathrm{deg}$, while $30 \mu \mathrm{m}$ shows the smallest value of $2.13 \mathrm{deg}$.

\subsection{Scatterometer Measurement}

Using the forward scatterometer, a series of scatterogram was aquired as the bacteria grew. The measurement was aquired as the incident laser beam waist was varied close to the colony diameter. The top row of Fig. 7 shows scatterograms from approximately 89,119 , and $144 \mu \mathrm{m}$ with $Z_{2}$ set around $8 \mathrm{~mm}$. Meanwhile, the bottom row displays the result for the colony diameters of approximately 193, 253, and $302 \mu \mathrm{m}$. For these sets, $Z_{2}$ was set to approximately $12 \mathrm{~mm}$ such that larger area of sensor was utilized to the resolve the multiple number of rings. The result clearly indicates the increase of the number of rings as the colony diameters increased, which were measured from phase contrast microscope.

\subsection{Colony Profile Measurement}

To accurately assess the $H_{0}, \mathrm{CDM}$ was used to acqure the profile, which was operated on the profile mode with 220 data points with a $5-\mu \mathrm{m}$ interval $^{23}$ in $Y$ direction. Figure 8 displays the colony profile measurement and corresponding diameter measured from the phase contrast microscope. Figure 8(a) consolidates the 1-D crossectional profile across the $H_{0}$ location for seven different colony diameters. The result indicates the following two obervations: (1) proporationality between $D$ and $H_{0}$, which is approximately 10:1 ratio, and (2) the

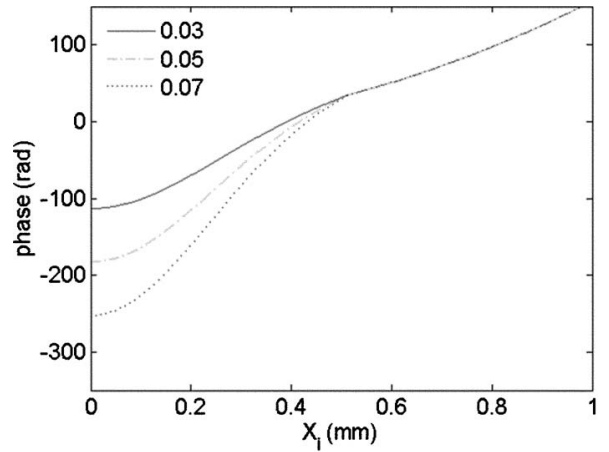

(a)

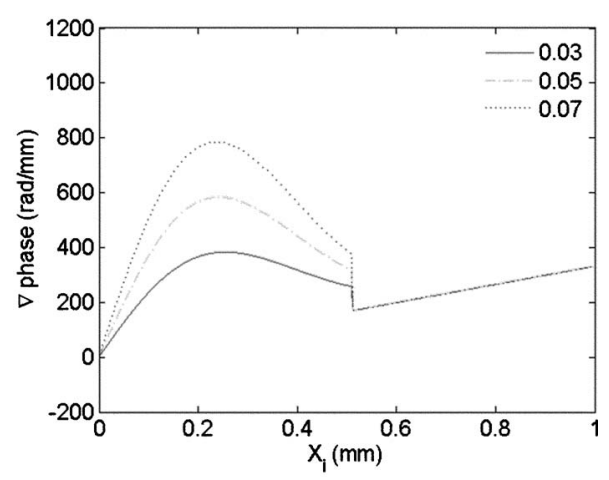

(b)

Fig. 6 (a) Phase and (b) derivative of phase of the wavefront after the incident beam passes the bacterial colony when the center thickness is $0.03,0.05$, and $0.07 \mathrm{~mm}$.

tailing edge profile was clearly captured for the most of the colony profiless, which supports our assumption on the Gaussian colony profile. Figures 8(b) and 8(c) show the phase contrast image and corresponding 2-D profiles from CDM in peudocolors.

To provide quantitative validations between theory and experiment, correlations between three different approaches were investigated: estimation from nematic crystal research, ${ }^{12,15}$ estimation from the proposed model, and the experimental result (Fig. 9). The red square indicates the es-

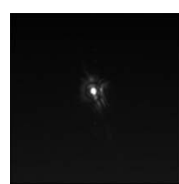

$83 \mu \mathrm{m}$

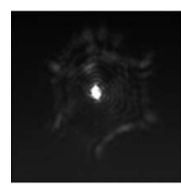

$193 \mu \mathrm{m}$

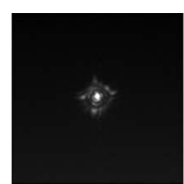

$119 \mu \mathrm{m}$

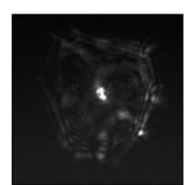

$252 \mu \mathrm{m}$

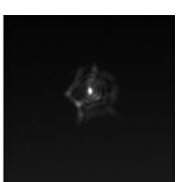

$144 \mu \mathrm{m}$

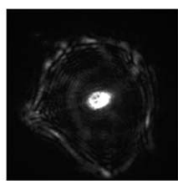

$302 \mu \mathrm{m}$
Fig. 7 Recorded forward scattering patterns against the colony diameter for Salmonella monteviedo. The diameter was varied from 89 to $302 \mu \mathrm{m}$. 


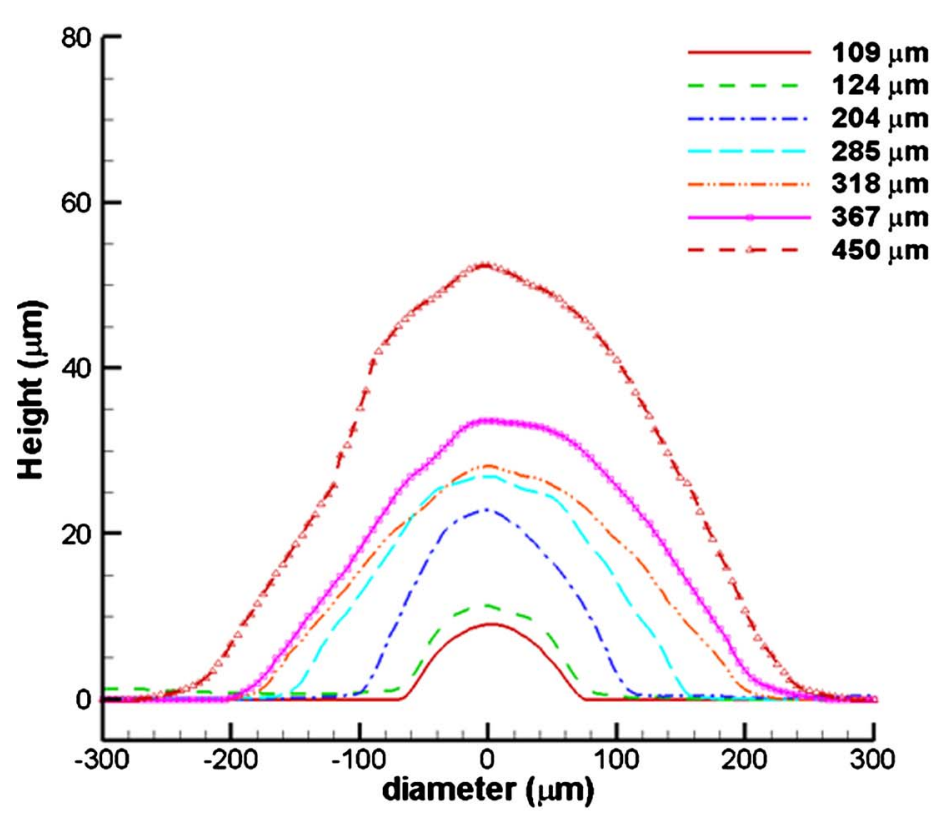

(a)
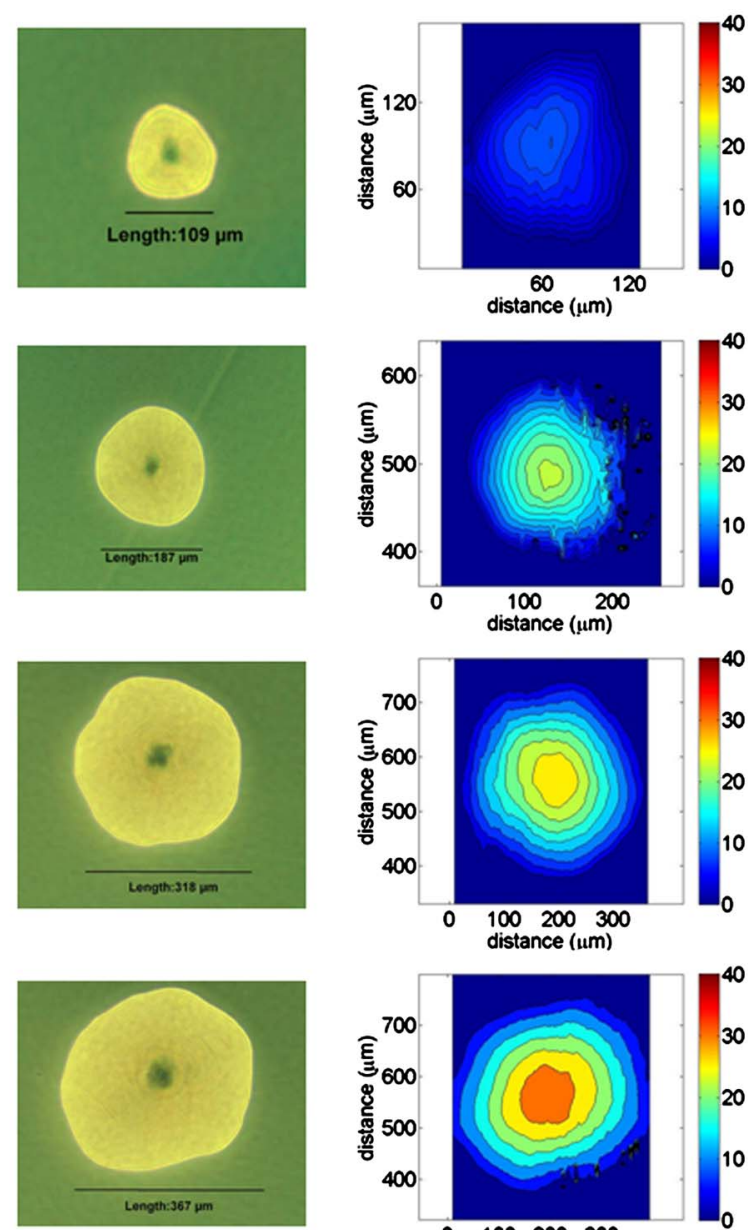

(b)

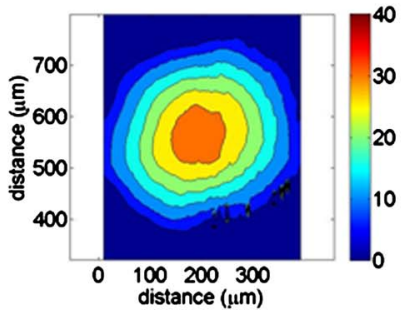

(c)

Fig. 8 Bacterial colony profile measurements: (a) the cross-sectional profile (vertical axis not to scale) for various size of bacterial colonies (109 to $450 \mu \mathrm{m}$ ), (b) the corresponding phase contrast images, and (c) the 2-D profile from CDM. (Note: second figure of (b) shows $187 \mu \mathrm{m}$ horizontal diameter but the vertical diameter is measured to be $204 \mu \mathrm{m})$.

timated ring counts [Fig. 9(a)] and maximum diffraction angle [Fig. 9(b)] from the $H_{0}$ measurement of the CDM, while the green triangle represents the those from the experiment (Fig. 7). Finally, the blue circle shows the estimation from the proposed diffraction model. In the experiment, the beam diameter was controlled to be similar to the colony diameter therefore Eq. (12) was applied to convert from $H_{0}$ to phase difference. To compare with our previous modeling effort, we fitted the measured colony profile with three different models and estimated their performance, which were Gaussian profile fit (Gfit), two-radius fit (TR-fit), and one-radius fit (OR-fit). The performance was measured by calculating the sum of the squared difference from the proposed model and the 1-D CDM profile across the peak location. Table 2 shows the results and indicates that the G-fit and TR-fit are comparable to each other and clearly capture the colony profile, while the OR-fit (single spherical profile) does not reflect the actual colony profile.

\section{Discussion}

\subsection{Maximum Diffraction Angle}

According to the results in Sec. 3, the maximum diffraction angle and the total number of rings are closely related to the bacterial colony morphology. These two parameters are important since they are directly measurable from the experiment and thus provide the phenotypic characteristics of the bacterial colonies. First, the maximum half diffraction angle is the result of the interference of two or more wavevectors pointing at the same direction. According to Eq. (13), the Fresnel diffraction pattern is the fast Fourier transform (FFT) of the input wavefront right after the wave passes through the bacterial colony. This accumulates all the light-matter interactions such as absorption, transmission, and reflection, which results in an overall phase modulation. Since the bacterial colony is modeled as a Gaussian profile, the wavefront emerging from the bacterial colony also resembles this Gaussian profile. Coupled with the radial phase effect, the overall wave- 
Bae et al.: Modeling light propagation through bacterial colonies and its correlation with forward scattering patterns

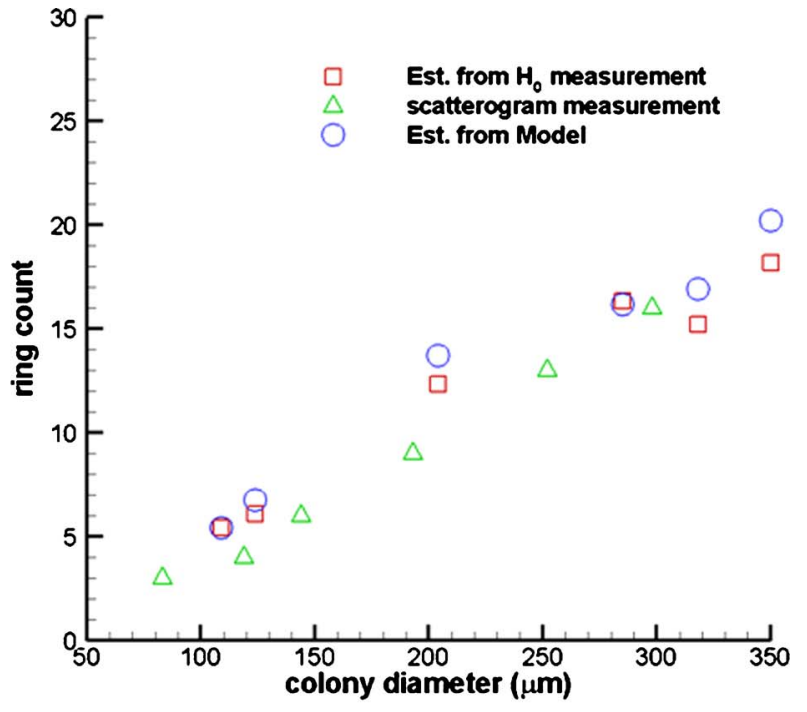

(a)

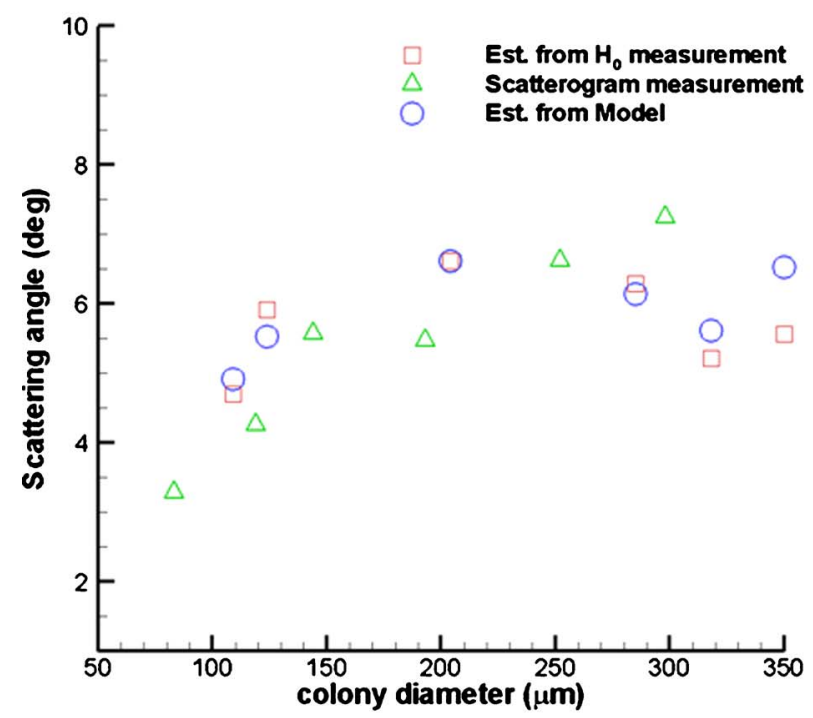

(b)

Fig. 9 Comparison of model and experimental result: (a) comparisons of the ring count versus colony diameter and (b) maximum half cone angle versus colony diameter. Red squares in (a) denote the estimated ring count from the $H_{0}$ measured from CDM using Eq. (16), while in (b) the derivative of phase is calculated from the profile and Eq. (17) was used for estimation. The green triangle represents the actual ring count from scatterograms (Fig. 7), while the blue circle shows the estimated ring count from the proposed model. (Color online only.)

front is determined by Eq. (14). Therefore, the maximum diffraction angle is governed by the direction of the wave vector, which is the normal vector to the emerging wavefront. This implies that the magnitude of the slope of the wavefront is the key factor to determining the maximum diffraction angle, a theory that was also suggested by other researchers using nematic crystals. ${ }^{12-16}$ Previous research from nematic LCs reported the equation for estimating the maximum half cone angle $\theta / 2_{\max }$ as,
Table 2 Comparison of the Gaussian (G), two-radius (TR), and oneradius $(\mathrm{OR})$ fits to the measured colony profiles.

\begin{tabular}{ccccr}
\hline & & \multicolumn{3}{c}{$\Sigma\left(P_{M}-\text { Fit }\right)^{2}$} \\
\cline { 3 - 5 } $\begin{array}{c}\text { Diameter } \\
(\mu \mathrm{m})\end{array}$ & $\begin{array}{c}\text { Height } \\
(\mu \mathrm{m})\end{array}$ & G-fit & TR-fit & \multicolumn{1}{c}{ OR-fit } \\
\hline 109 & 9.04 & 10.21 & 21.42 & 6.39 \\
124 & 11.28 & 29.84 & 25.43 & 564.49 \\
204 & 22.83 & 150.65 & 128.42 & 2181.03 \\
285 & 26.95 & 209.81 & 263.18 & 4808.79 \\
318 & 28.19 & 145.64 & 265.87 & 1387.36 \\
367 & 33.64 & 275.18 & 341.80 & 3436.84 \\
421 & 52.37 & 700.19 & 2993.44 & $14,350.91$ \\
\hline
\end{tabular}

$$
\theta / 2_{\max } \cong \frac{1}{k}\left(\frac{\mathrm{d} \Delta \Phi}{\mathrm{d} r}\right)_{\max },
$$

where $k$ is the wave number. ${ }^{20}$ Figure 6(b) provides the derivative of the phase profile based on model profile of Fig. 6(a). The maximum half cone angle of the total number of rings (when $D=1.024$ ) for the series of $H_{0}$ for $0.03,0.05$, and $0.07 \mathrm{~mm}$ as $2.13,3.21$, and 4.42 , which agrees with 1.91 , 3.08, and 4.25 from Eq. (18).

\subsection{Number of Diffraction Rings}

The total number of rings is another parameter closely related to the colony morphology. As shown in Fig. 5, the diffraction rings are dependent on the center thickness and are not dependent on the the colony diameter. This is because the optical path difference (OPD) increases as the $H_{0}$ is increased. To help clarify this argument, we provide a schematic diagram in Fig. 10, which represents the wavefront from the bacterial colony. Since the wavefront captures the colony profile, the emerging wavefront is also a Gaussian profile. Since the dif-

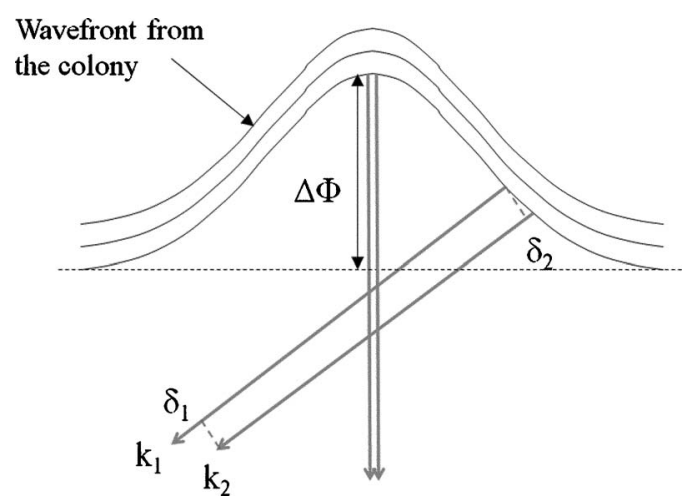

Fig. 10 Schematic diagram for understanding the interference pattern generated by the emerging wavefront: $\Delta \Phi$ represents the phase lag from the colony center thickness $H_{0}, \mathbf{k}_{1}$ and $\mathbf{k}_{2}$ represent the wave vectors, and $\delta_{1}$ and $\delta_{2}$ represent the OPD difference of these two wave vectors. 
fraction peaks and valleys are generated from the interference of two or more wave vectors, we can relate the phase lag $(\Delta \Phi)$ to the number of rings. Since the wave vectors $\mathbf{k}_{1}$ and $\mathbf{k}_{2}$ will interfere in at the far field, their OPD determines whether we observe a peak or valley, depending on constructive and destructive interference. As shown in Fig. 10, this OPD is determined from $\delta_{1}$ and $\delta_{2}$, which decreases when the bacterial colony profile gets flatter $\left(H_{0}\right.$ is decreased). Therefore, we can infer that the increase of $H_{0}$ results in a larger OPD and creates a larger number of diffraction rings. This conclusion coincides with conclusions drawn from nematic crystal research, ${ }^{12,15}$ where the number of rings $N_{\text {ring }}$ was estimated as

$$
N_{\text {ring }} \cong \frac{\Delta \Phi}{2 \pi} .
$$

This fact is confirmed when we compare the estimation of Eq. (19) with our result in Sec. 3. Figure 5(b) provides the mean value of the total number of rings for the series of $H_{0}$ for 0.03 , 0.05 , and $0.07 \mathrm{~mm}$ as $29.0,39.8$, and 50.9 , which is close to 28, 40, and 52 when estimated from Eq. (19) and Fig. 6(a). For example, when $H_{0}$ is $0.03 \mathrm{~mm}, \Delta \Phi$ is computed to be 186 (by substracting the phase at $X_{i}=0.8$ and $X_{i}=0$ since the Gaussian incident beam diameter is $1.6 \mathrm{~mm}$ for $w_{b}=0.5$ ), while Eq. (19) gives approximately 182.

\subsection{Experimental Verification}

The proposed Guassian colony profile model was compared with experiment and other models of Eqs. (18) and (19). Figure 9(a) shows the comparision of the number of rings versus the colony diameter up to the $350-\mu \mathrm{m}$ range, where the predicted and measured ring counts agree well. Some differences are still observed, though especially for the smaller colonies where the bright central spot lowers the accuracy in the experimental ring counts due to the limited dynamic ranges of the CMOS sensor. Figure 9(b) displays a similar comparison for a maximum diffraction angle. Both the proposed model and Eq. (19) predict similar results and the experimental measurement matched within a reasonable range of angles. A possible error source could arise from the difference between the Gaussian fit and the real colony and the position measurement of $Z_{2}$, since the scattering angle masurement is sensitive to the colony-image plane distance. The result from Table 2 clearly shows the possibility of using a Gaussian profile to analyze the optical characteristics of the colony. Although the TR-fit model showed comparable accuracy with the G-fit model (except for diameters larger than $350 \mu \mathrm{m}$ ), the proposed model requires only the $H_{0}$ and $D$ values, while the TR-fit requires iterative optimizations of three different spherical profiles to accurately model the profile. Thus, we can directly estimate the morphological characteristics of the bacterial colony based on observation of the diffraction pattern. This explanation can be applied to the previous results from the timeresolved scattering pattern analysis. ${ }^{3}$ Our theory argues that vertical growth velocity is approximately equal to lateral growth velocity in the lag and logarithmic bacterial growth phase, which results in a wide diffraction pattern since the colony is narrow and tall. On the other hand, once the colony reaches the stationary point, vertical growth is slowed com- pared to lateral growth; hence, the diffraction pattern tends to shrink since the colony shape becomes wider and shorter.

\section{Conclusion}

Scalar diffraction theory was applied to model a bacterial colony as optical amplitude and phase modulators. Assuming a Gaussian profile for the colony shape, two main morphological characteristics of center thickness and colony diameter were calculated to correlate with the corresponding diffraction pattern. The results indicated that the maximum diffraction angle is dependent on the magnitude of the slope of the wavefront emerging from the bacterial colony, while the total number of rings is dependent on the center thickness of the bacterial colony. These results verify a fast method of understanding the bacterial colony morphology, which can be related to the understanding and classification of bacterial species.

\section{Acknowledgments}

This research was supported through a cooperative agreement with the Agricultural Research Service of the U.S. Department of Agriculture Project No. 1935-42000-035 and the Center for Food Safety and Engineering at Purdue University.

\section{References}

1. E. Bae, P. P. Banada, K. Huff, A. K. Bhunia, J. P. Robinson, and E. D. Hirleman, "Biophysical modeling of forward scattering from bacterial colonies using scalar diffraction theory," Appl. Opt. 46(17), 3639-3648 (2007)

2. P. P. Banada, S. L. Guo, B. Bayraktar, E. Bae, B. Rajwa, J. P. Robinson, E. D. Hirleman, and A. K. Bhunia, "Optical forward-scattering for detection of Listeria monocytogenes and other Listeria species," Biosens. Bioelectron. 22(8), 1664-1671 (2007).

3. E. Bae, P. P. Banada, K. Huff, A. K. Bhunia, J. P. Robinson, and E. D. Hirleman, "Analysis of time-resolved scattering from macroscale bacterial colonies," J. Biomed. Opt. 13(1), 014010 (2008).

4. P. P. Banada, K. Huff, E. Bae, B. Rajwa, A. Aroonnual, B. Bayraktar, A. Adil, J. P. Robinson, E. D. Hirleman, and A. K. Bhunia, "Labelfree detection of multiple bacterial pathogens using light-scattering sensor," Biosens. Bioelectron. 24(6), 1685-1692 (2009).

5. M. A. Bees, P. Andresen, E. Mosekilde, and M. Givskov, "The interaction of thin-film flow, bacterial swarming and cell differentiation in colonies of Serratia liquefaciens," J. Math. Biol. 40(1), 27-63 (2000).

6. R. Bernard, M. Kanduser, and F. Pernus, "Model-based automated detection of mammalian cell colonies," Phys. Med. Biol. 46(11), 3061-3072 (2001).

7. G. Corkidi, R. Diaz-Uribe, J. L. Folch-Mallol, and J. Nieto-Sotelo, "COVASIAM: an image analysis method that allows detection of confluent microbial colonies and colonies of various sizes for automated counting," Appl. Environ. Microbiol. 64(4), 1400-1404 (1998).

8. P. R. Barber, B. Vojnovic, J. Kelly, C. R. Mayes, P. Boulton, M. Woodcock, and M. C. Joiner, "Automated counting of mammalian cell colonies," Phys. Med. Biol. 46(1), 63-76 (2001).

9. J. Marotz, C. Lubbert, and W. Eisenbeiss, "Effective object recognition for automated counting of colonies in Petri dishes (automated colony counting)," Comput. Methods Programs Biomed. 66(2-3), 183-198 (2001).

10. N. Bai, E. Bae, A. Aroonnual, A. K. Bhunia, J. P. Robinson, and E. D. Hirleman, "Development of a real-time system of monitoring bacterial colony growth and registering the forward-scattering pattern," in Sensing for Agriculture and Food Quality and Safety, Proc. SPIE 7315, 73150Z-73158 (2009).

11. F. Bloisi, L. Vicari, F. Simoni, G. Cipparrone, and C. Umeton, "Selfphase modulation in nematic liquid-crystal films-detailed measurments and theoretical calculations," J. Opt. Soc. Am. A 5(12), 2462 2466 (1988).

12. S. H. Chen, J. Y. Fan, and J. J. Wu, "Novel diffraction phenomenon 
with the first order Fredericksz transition in a nematic liquid crystal film," J. Appl. Phys. 83(3), 1337-1340 (1998).

13. S. D. Durbin, S. M. Arakelian, and Y. R. Shen, "Laser-induced diffraction rings from a nematic-liquid-crystal film," Opt. Lett. 6(9), 411-413 (1981).

14. I. C. Khoo, J. Y. Hou, T. H. Liu, P. Y. Yan, R. R. Michael, and G. M. Finn, "Transverse self-phase modulation and bistability in the transmission of a laser-beam through a nonlinear thin-film," J. Opt. Soc. Am. A 4(6), 886-891 (1987).

15. K. Ogusu, Y. Kohtani, and H. Shao, "Laser-induced diffraction rings from an absorbing solution," Opt. Rev. 3(4), 232-234 (1996).

16. R. P. Pan, S. M. Chen, and C. L. Pan, "A quantitative study of the far-field laser-induced ring pattern from nematic liquid-crystal films," Chin. J. Physiol. 30(4), 457-466 (1992).

17. E. Santamato and Y. R. Shen, "Field-curvature effect on the diffraction ring pattern of a laser-beam dressed by spatial self-phase modu- lation in a nematic film," Opt. Lett. 9(12), 564-566 (1984).

18. M. Sheikbahae, A. A. Said, T. H. Wei, D. J. Hagan, and E. W. Vanstryland, "Sensitive measurement of optical nonlinearities using a single beam," IEEE J. Quantum Electron. 26(4), 760-769 (1990).

19. A. Shevchenko, S. C. Buchter, N. V. Tabiryan, and M. Kaivola, "Creation of a hollow laser beam using self-phase modulation in a nematic liquid crystal," Opt. Commun. 232, 77-82 (2004).

20. J. J. Wu, S. H. Chen, J. Y. Fan, and G. S. Ong, "Propagation of a Gaussian-profile laser-beam in nematic liquid-crystals and the structure of its nonlinear diffraction rings," J. Opt. Soc. Am. A 7(6), 11471157 (1990).

21. J. W. Goodman, Introduction to Fouirier Optics, McGraw-Hill, New York (1996).

22. A. E. Siegman, Lasers, University Science Books, Sausalito (1986).

23. Keyence-Corporation, "Surface scanning laser confocal displacement meter LT-9001 series," Keyence Corporation, Tokyo (2006). 